\section{JOURNAL REVIEW}

\section{Women's understanding of gynaecological cancers and gynaecological cancer screening}

Hawkins NA, Cooper CP, Saraiya M, et al. Why the Pap test? Awareness and use of the Pap test among women in the United States. J Womens Health (Larchmt) 2011;20:511-515.

Cooper CP, Polonec L, Gelb CA. Women's knowledge and awareness of gynecologic cancer: a multisite qualitative study in the United States. J Womens Health (Larchmt) 2011;20:517-524.

Gynaecological (or gynecologic) cancer refers to cancer of the reproductive organs including the cervix, ovaries, uterus, vulva and vagina. The only screening currently recommended for a gynaecological cancer is cervical screening, with most cases of cervical cancer occurring in unscreened women. For other gynaecological cancers early diagnosis is likely to increase survival outcomes. ${ }^{1}$

In April 2011, the Journal of Women's Health published two papers that used different methodologies to explore women's general understanding of gynaecological cancers and the purpose of cervical screening. Both studies were carried out in the USA as part of the development of the Centers for Disease Control and Prevention 'Inside Knowledge' campaign (which aims to raise awareness of the five main types of gynaecological cancer). The message that comes across strongly in both papers is that women have a poor understanding about these types of cancer and of the purpose of cervical screening (described in both papers as the Pap test). In the first paper, Hawkins et al. surveyed nearly 3000 women, asking them what a Pap test is used "to check for", with responses made by selecting from a predefined list. These women had previously taken part in an unrelated survey and had high incomes and education levels. Therefore, they are not representative of the US population, although the data were weighted to take account of this. In addition over $90 \%$ of the sample had previous experience of cervical screening. However, only $17 \%$ knew that the Pap test screened exclusively for cervical cancer. Many of the women thought the Pap test detected vaginal (41\%), ovarian (37\%) or uterine cancer (34\%), as well as human papillomavirus (HPV) (31\%), other sexually transmitted infections (34\%) and yeast infections (30\%). These findings raise concerns about women's understanding of the purpose of cervical screening.

In the second paper, Cooper et al. used a different methodology to explore women's understanding of gynaecological cancers, carrying out 15 focus groups with 132 women across different locations in the USA and analysing the data using an inductive (data-driven) thematic approach. Most of the women were familiar with cervical, ovarian or uterine cancer, but had not heard of vulval or vaginal cancer. They were also unfamiliar with the term 'gynaecological cancer' and there was some confusion as to what this 'category' would and would not include (e.g. breast cancer). Most women thought the Pap test screened for multiple conditions, with some describing it as an "all-inclusive" test covering multiple cancers and other conditions, although the strength of this belief varied. The paper also suggested gaps in women's knowledge of risk factors and symptoms. Some were aware that cervical cancer was caused by HPV, and some suggested vaginal bleeding as a symptom of cervical cancer, but women were unaware of the risk factors or symptoms for other gynaecological cancers. This is consistent with poor recall of the symptoms for cancer more generally, as assessed by the Cancer Awareness Measure in the UK. ${ }^{2}$

The findings from these two papers have several implications. Despite most women having experience of cervical screening, their understanding of its purpose was poor. This suggests that knowledge of screening may not be a prerequisite for attendance, but given the move towards informed decision-making in relation to screening, helping women to understand the test they are having should still be considered important. What is not discussed in either of these papers is the fact that the Pap test is actually designed to detect precancerous changes and is not primarily a test for cancer. This could be an additional concern given the potential anxiety associated with misunderstanding an abnormal result.

Misconceptions about the purpose of screening may also lead to false reassurance regarding other gynaecological cancers for which women may still be at risk. This sense of reassurance, alongside women's lack of awareness about gynaecological cancers, and their signs and symptoms, may result in increased delay in seeking medical attention. Public information campaigns designed to address awareness and misperceptions about gynaecological cancers are therefore important to increase informed choice about cervical screening, to ensure the accuracy of women's risk perceptions and to increase gynaecological cancer survival through early diagnosis.

\section{Reviewed by Laura A V Marlow}

Cancer Research UK Postdoctoral Fellow, Department of Epidemiology \& Public Health, Health Behaviour Research Centre, University College London, London, UK

Competing interests None.

J Fam Plann Reprod Health Care 2011;37:256. doi:10.1136/jfprhc-2011-100157

\section{REFERENCES}

1. Thomson CS, Forman D. Cancer survival in England and the influence of early diagnosis: what can we learn from recent EUROCARE results? Br J Cancer 2009;101(Suppl. 2):S102-S109

2. Robb K, Stubbings S, Ramirez A, et al Public awareness of cancer in Britain: a population-based survey of adults. Br J Cancer 2009;101(Suppl. 2):S18-S23. 